\title{
A Study on Regulatory Reform of China's Taxi Industry in the era of Internet Plus
}

\author{
Yanfang $\mathrm{Li}^{1,}$, , Peihong Chen ${ }^{2, \mathrm{~b}}$ \\ ${ }^{1}$ School of Economics and Management, Beijing Jiaotong University, Beijing, China \\ ${ }^{2}$ School of Economics and Management, Beijing Jiaotong University, Beijing, China \\ a14120515@bjtu.edu.cn, bphchen@bjtu.edu.cn
}

\begin{abstract}
Keywords: taxi; regulatory reform; Internet Plus; car-hailing services.
Abstract. For a long time, the taxi industry is heavily enforced with inefficient entry and price regulation in China, which has restricted the development of taxi market and accumulated many problems. Under this circumstance, new business model of "Internet + transportation" has provided an excellent opportunity for the reform of traditional taxi industry. Meanwhile, it puts forward requirement of improving taxi industry management capability. Thus, the government shall seize this opportunity to reconstruct taxi industry and regulatory systems. To achieve this goal, participants should firstly identify the problems existing in the traditional taxi industry regulation, as well as the impact of Internet Plus.
\end{abstract}

\section{Introduction}

In October 2015, the ministry of transport released draft rules on the management of online car-hailing services. The regulations intend to integrate the online car-hailing services into the taxi management system and raise the access threshold. However, there are also criticisms of the draft rules because they may have a negative impact on not only current online car-hailing services, but also the sharing economy based on the Internet. The new rule goes against the sharing economy and is not environmentally friendly. Some people have even commented that the regulations turn "Internet Plus" into "Internet Minus".

As online car-hailing service is becoming increasingly popular in many cities, it is necessary and reasonable to make up rules to manage the industry, because it is closely related to the interests and safety of users. Meanwhile, as car-hailing services have conflicts of interests with the taxi industry, it is the administrator's responsibility to mitigate contradictions among different stakeholders for the sake of social stability. Thus, how to innovate the regulatory systems of the taxi industry will be a major issue to be solved.

\section{Literature References}

Many studies have been published in relation to the car-hailing services sector at home and abroad. Different scholars have different directions and methods, mainly including sharing economy, traditional taxi industry regulations, platform behavior, etc. Birdsall and Michelle pointed out that the growing acceptance of the sharing economy concept combined with rapid digital technology advances are enabling significant strides in the implementation of multimodal transportation solutions that promise to ease congestion and reduce demand on systems that can no longer afford physical capacity increases [1]. Mary Anne M. Gobble shared her views on how digital innovation is regulated under new economic conditions. She thought as privacy concerns grow and more trust-based and data-centric apps come to market, thinking about these questions may become a critical element of the business model. If these apps can't capture consumers' trust and allay their fears, customers will go elsewhere to providers who can make them feel comfortable [2]. Lixia Zhou believed that on the one hand, Chinese government should strictly stimulate the access conditions of domestic car-hailing services, combine the administrative supervision with the effective industry regulation and strengthen the constraints of corresponding laws and regulations; on the other hand, given the car-hailing services' unique business model and operating characteristics, the regulation 
can't completely copy the traditional taxi industry regulations [3]. Chaohe Rong and Xuecheng Wang found that bigoted dichotomy between public transport and private transport had set many limitations for the policy-makers of taxi industry. Thus, the regulators should identify the concepts of taxi and other transportation, define the nature of taxi industry and then clarify its function and status [4].

\section{The limitations of traditional taxi industry regulation in China}

The nature and type of taxi services. Taxi is the car which provides services to passengers and charges for passengers according to the mileage and time. Taxi services can be divided into three broad categories: stand segment, cruising segment and telephone-booking segment. In the stand segment, there are designated places where taxi can wait for passengers and vice versa. Taxis and customers are usually forming queues at some fixed stop and customers must walk to the nearest taxi stop. In the cruising market, clients hail a cruising taxi on the street. There is uncertainty about the waiting time and the quality/fare of the service customers will find. Customer mustn't walk to the taxi stop. In the telephone-booking market, consumers telephone a dispatching center asking for an immediate taxi service or for a later taxi service. Only in this kind of market consumers can choose between different service providers or companies.

The forms of taxi market regulation. The taxi industry is one of the most heavily regulated modes of transport in China. The argument for regulation in the taxi industry is predominantly based around avoiding market failure by developing regimes to maintain and enforce standards to taxi drivers and their companies. There are mainly two kinds of regulatory model: one is entry regulation, which strictly limits the number of taxi, especially the individual taxis; the other is fare control, which limits the price of taxi. More specially, fares are usually regulated by fixing a maximum price and regulating the way that fares are applied to customers (per time and per distance, etc.). What's more, most of the entry regulations are done simply by freezing the number of taxi licenses.

Possible problems existing in the taxi industry. Under such a strict control, the closed conservation of taxi industry has become a quite serious and common problem.

Firstly, the regulation of license leads to the shortage of whole taxi supply market, which can't meet the increasing demand. For example, in Beijing from 2004 to 2015, the population of permanent residents increased from 14.92 million to 21.15 million, but from 2003 to 2012, the number of taxis only increased from 65000 to 66000 . Since 2012, due to the limited road resources and requirements of energy conservation, the number keeps in 66000. Apparently, the existing number of taxis can't meet the demand of the resident population, making it a difficulty to take a taxi, especially in some transportation junctions, commercial centers and hospitals.

Secondly, the fares control leads to the loss of total social welfare. Because of the existence of asymmetric information between the government and the market, the government can't accurately measure equilibrium price, which makes the regulated price being inconsistent with the equilibrium price. On the one hand, when the regulated price is higher than the market equilibrium price, consumers are the biggest victims of the price control. High prices discourage passengers, and the empty of the taxi also causes a lot of waste of resources. On the other hand, when the regulated price is lower than the market equilibrium price, drivers are the biggest victims of the price control. Low fares lead to the shortage of legitimate taxi supply and the prevalence of unlicensed taxi.

\section{The impact of "Internet +" to the traditional taxi industry}

The meaning and influence of Internet Plus. To put it simply, Internet Plus can be interpreted as "Internet + various traditional industries", which takes advantage of the information and communications technology as well as the Internet platform to integrate the Internet and traditional industries, changing the ordinary people's lifestyles and creating a new modal of development. As a result of practice of the developing Internet techniques, Inter Plus stands for a new advanced productivity and promotes an ever-expanding economic type, bringing a strong vitality to social economic entity. Car-hailing service, a new "Internet +taxi industry" business model, which helps 
passengers get taxis more easily, has developed rapidly in China, posing a challenge to traditional taxi industry.

The nature and influence of car-hailing service. As an advanced type of taxi appears in everyday life, the car-hailing service provides a service similar to the telephone-booking service but particularly uses the mobile application. On the one hand, with lower transaction costs, higher transparency, better customer experience and other advantages, car-hailing service is not only to provide users with more travel options, changing the existing structure of urban transportation, but also greatly mobilize the social resources, making the entry barriers broken and introducing competition into the field of the taxi. On the other hand, car-hailing service platforms enable a much larger number of individuals to contract directly for goods and services, and this decentralization fundamentally alters the regulatory landscape. In China, as more and more Internet enterprises to enter, by introducing new forms of service and increasing the intensity of subsides, the major platforms are making the Internet-based business model grow fast in more than 200 cities.

The case of Didi Kuaidi and Uber. The rise of car-hailing services and Internet companies has rightly served as an alarm call to both traditional taxi operators and the industry regulators. For example, Didi Chuxing, China's largest car-hailing app, handled 1.43 billion rides in 2015, followed by Uber, the world's most popular car-hailing app, which had 1 billion rides for the same year. According to the market prediction, Didi Kuaidi has about 80 percent of the car-hailing service market and Uber has about 10 percent.

Didi Chuxing, formerly Didi Kuaidi, is a Chinese transportation network company, which offers a full range of commuting options in China via smartphone applications. The company is the result of the merger between taxi-hailing firms Didi Dache (backed by Tencent Holding Limited) and Kuaidi Dache (backed by Alibaba Group). Offering a broad range of mobile technology-based transportation options across over 400 major Chinese cities, including taxi hailing, private car hailing, Hitch (social ride-sharing), Chauffeur, Didi Bus, Didi Test Drive, and Didi Enterprise Solutions, Didi Kuaidi is already the world's largest transportation service platform in terms of the number of rides and customers served each day.

Uber, an American multinational online transportation network company, allows consumers with smartphone to submit a trip request which is then routed to Uber drivers who use their own cars. Now, the service is available in over 66 countries and 449 cities worldwide. In 2014, Uber launched a nonprofit car- hailing service in China called People’s Uber. On the operating mode, Uber mainly provides platform for private cars to serve consumers, which violates the relevant provisions of China that car-hailing services offered by private drivers without a taxi license will be illegal.

\section{The reform of the regulatory system under the new forms}

Controversial draft rules. The draft rules, which released by the ministry of transport in 2015, are intended to properly regulate the booming sector, but are set to cast a shadow over the existing operations of major players such as the Chinese company Didi Kuaidi and its US rival Uber. According to the rules, private car-owners must register their vehicles as commercial taxis, meaning that their cars will be written off in eight years, regardless of their actual condition, if they become car-hailing service providers. Obviously, these draft rules aim to place the nontraditional taxis under traditional management, and fail to meet the increasing demand for the sharing economy, which can significantly reduce social costs by breaking the boundaries between private and public resources.

Policy recommendations. The taxi industry is particularly highly regulated, and there are perpetual calls to lower to barriers to entry. Car-hailing companies like Didi Kuaidi and Uber have disrupted lucrative existing monopolies, which will give the regulators a new opportunity and motivation to readjust the regulatory systems and methods. What's more, the current rules that gouge one group to benefit another also need to be reformed.

First of all, the traditional taxi services and the online car-hailing services should be coordinated with relevant professional departments on classified management. On the one hand, regulators should specify that the traditional taxi services can still use the previous operating mode such as in cruising 
and telephone-booking market, and continue to implement the government pricing. One the other hand, the online car-hailing services can only use smartphones and taxi apps to accept orders, and implement the dual regulation of government guidance and market regulation to adjust the price. Thus, both the traditional taxi operation model and the new model can coexist and build the "Internet + taxi” new format.

Secondly, the car-hailing service platforms should be the key of the regulation. The platforms only emphasize their matching function, regardless of responsibilities they should take on, which causes concerns over their unconstrained expansion behavior through the cash-burning fight. Thus, the regulators have to make the car-hailing service providers such as Didi Kuaidi and Uber hold major responsibility for any disputes or safety accidents during the operation. Then, platform companies will consciously improve the threshold of the qualifications and strictly control the registered vehicles and drivers, trying to avoid excessive risk.

Thirdly, taking advantage of the platform to implement the regulation will be more effective. Platforms can be better than governments at spotting stalkers, running background checks on vehicles and drivers, and responding quickly to conflicts among registrants. That is to say, platforms are closer to the action and they have an incentive to look after their whole network. For example, Didi said it would cooperate with departments concerned to strictly follow laws and regulations. The company also promised to partner with local governments to establish a data platform to monitor cars and drivers on its platform.

\section{Summary}

In the face of the complex situations, any rush shock-mounted reform may cause the whole industry unstable and previous efforts may also be in vain. This evolving and growing market requires close collaboration among consumers, government and platforms to foster innovation and ensure access to viable insurance coverage and proactive risk management. That the car-hailing services are playing a key role in the transportation industry is a natural result of Internet Plus and sharing economy. In this sense, the regulators should open the door of taxi industry and introduce more competition, forcing the traditional taxi participants to improve their efficiency, provide better services and change the present situation of the taxi market.

\section{Acknowledgments}

This work was supported by the World Bank Loan Project (Loan No.8346-CN): the urban traffic demand management policy research of Mudanjiang (Contract No.mjj016). At last, I'd like to express my sincere gratitude to my tutor, professor Chen.

\section{References}

[1] Birdsall, Michelle: Car-sharing in a Sharing Economy, Institute of Transportation Engineers. ITE Journal, Vol.84 (2014), p. 37-40.

[2] Mary Anne M. Gobble: Regulating Innovation in the New Economy, Research Technology Management, Vol.58 (2015), p. 62-64.

[3] Lixia Zhou: The Thoughts of how to Regulate the Domestic Taxi Apps, Price Theory and Practice, (2015), p. 21-24.

[4] RONG Chaohe, WANG Xuecheng: Identify the nature of online booked vehicle and promote regulatory reform of taxi industry, China Transportation Review, Vol.38, (2016), p. 4-10. 